\title{
A randomized trial on the application of a nurse-led early rehabilitation program after minimally invasive lumbar internal fixation
}

\author{
Wei He", Qingsi Wang", Jiang Hu", Shu Lin, Kun Zhang, Fei Wang, Chunyi Xu, Fangjia Li, Jingrong Xiao, \\ Xiaoxue Li, Fang Tang \\ Department of Orthopedics, Sichuan Provincial People's Hospital-Sichuan Academy of Medical Sciences, Chengdu, China \\ Contributions: (I) Conception and design: F Tang, X Li; (II) Administrative support: C Xu; (III) Provision of study materials or patients: F Li, J Xiao; (IV) \\ Collection and assembly of data: W He, Q Wang, J Hu; (V) Data analysis and interpretation: S Lin, K Zhang, F Wang; (VI) Manuscript writing: All \\ authors; (VII) Final approval of manuscript: All authors. \\ \#These authors contributed equally to this work. \\ Correspondence to: Fang Tang; Xiaoxue Li. Department of Orthopedics, Sichuan Provincial People's Hospital, 32 West Second Section of the First \\ Ring Road, Qingyang District, Chengdu 610072, China. Email: 754487934@qq.com; 1247549722@qq.com.
}

Background Lumbar degenerative disease (LDD) is a narrowing of the spinal canal and intervertebral
foramina caused by aging and degeneration of lumbar spine tissue. Minimally invasive internal lumbar
spine fixation is emerging in the treatment of LDD. However, no standard early rehabilitation protocol for
orthopedic robot-assisted minimally invasive internal lumbar spine fixation exits. To investigate the effect of
a nurse-led early rehabilitation program in the postoperative recovery of patients with lumbar degenerative
lesions undergoing orthopedic robot-assisted minimally invasive lumbar internal fixation. Methods: Eighty patients with minimally invasive orthopedic robot-assisted lumbar degenerative lesions admitted to our hospital between January 2019 and January 2021 were recruited to this study. The patients were randomly allocated to a control group $(n=40)$, involving conventional care, and an observation group ( $\mathrm{n}=38$; 40 initially and 2 later excluded) including a nurse-led early rehabilitation program added on the basis of the control group. The primary outcomes were the general postoperative conditions, scores of daily living ability, the degree of low back pain and functional recovery. Participants were also compared in terms of their compliance with the care regimen and the incidence of complications.

Results: Participants in the observation group had a significantly shorter first time on the floor after surgery $(\mathrm{P}<0.001)$ and shorter hospital stay $(\mathrm{P}=0.003)$. Meanwhile, participants in the observation group had higher Barthel index $(\mathrm{BI})$ scores $(\mathrm{P}=0.039)$, lower visual analogue scale (VAS) scores $(\mathrm{P}=0.028)$, and Oswestry disability index $(\mathrm{ODI})$ scores $(\mathrm{P}=0.002)$ at 3 days postoperatively, and there was no difference in the three scores between the two groups at 1 month postoperatively (all $\mathrm{P}>0.05$ ). The compliance of participants in the observation group was significantly higher than that of the control group $(\mathrm{P}<0.001)$. Participants in the observation group were less likely to experience constipation $(\mathrm{P}=0.043)$ and bloating $(\mathrm{P}=0.012)$ within 1 month after surgery.

Conclusions: Implementation of a nurse-led early rehabilitation program in patients undergoing orthopedic robotic-assisted minimally invasive treatment of lumbar degenerative lesions can significantly improve patient compliance, significantly shorten postoperative flooring and hospitalization time, reduce the incidence of gastrointestinal adverse events, and accelerate postoperative recovery.

Trial Registration: Chinese Clinical Trial Registry ChiCTR2100048698.

Keywords: Nurse-led; orthopedic robot; lumbar degenerative disease (LDD); early rehabilitation

Submitted Jul 19, 2021. Accepted for publication Sep 09, 2021.

doi: 10.21037/apm-21-2294

View this article at: https://dx.doi.org/10.21037/apm-21-2294 


\section{Introduction}

Lumbar degenerative disease (LDD) refers to the narrowing of the spinal canal and intervertebral foramina caused by aging and degeneration of the lumbar spinal tissues. It is mainly classified into lumbar disc herniation (LDH), lumbar spinal stenosis (LSS), and lumbar spondylolisthesis (LS) $(1,2)$. Currently, the clinical treatment of LDD is mainly conservative, with the main goal of relieving low back and leg pain, restoring spinal stability, and preventing further aggravation of neurological dysfunction; when conservative treatment is ineffective, surgical treatment is required (3). The traditional procedure is pedicle screw fixation, which is effective in restoring spinal stability and improving spinal fusion rates and is the cornerstone of treatment for patients with severe LDD (4). However, pedicle screw fixation requires extensive tissue dissection, is prone to nerve and vascular damage, and has the disadvantages of poor postoperative recovery and secondary dysfunction (5). Additionally, this procedure requires a high level of skill and clinical experience of the surgeon in charge.

With the popularization of the concept of orthopedic precision treatment, the third-generation orthopedic robot "Tiangui" is gradually being incorporated into clinical practice and provides a more precise treatment solution for orthopedic diseases with its small incision, safety, precision, and fatigue resistance (6). The advantages of the "Tiangui" robot are reflected in both preoperative and intraoperative aspects. Preoperatively, it can provide personalized intelligent planning according to the patient's specific situation; intraoperatively, it can provide precise image guidance along with the surgery, thus reducing intraoperative injuries, improving the quality of surgery, and reducing chronic back pain for the patient after surgery (7). The minimally invasive lumbar internal fixation is surgery approach with smaller incisions, less blood loss, quicker return to daily activities, and increased visualization (5). With the aid of the "Tiangui" robot, minimally invasive lumbar spinal internal fixation is gradually emerging in the treatment of LDD.

With the advancement of medical concepts and development of minimally invasive orthopedic robotic surgery, some more precise requirements for the provision of postoperative care have been stipulated. At the present stage, the traditional care model is still adopted for patients after minimally invasive lumbar internal fixation, where patients are usually first out of bed 1-7 days after surgery and after the drainage tube is removed; however, this traditional care model can no longer match the rapid postoperative recovery enabled by minimally invasive surgery (8). Although as yet there is no standard early rehabilitation protocol for orthopedic robot-assisted minimally invasive lumbar internal fixation, early rehabilitation exercises have been reported in the literature to accelerate patients' postoperative recovery (9). A previous randomised clinical studies of 60 patients undergoing traditional lumbar fusion for degenerative lumbar disease have revealed the integrated programme of prehabilitation and early rehabilitation in spine surgery is more cost-effective compared to standard care programme alone (10). A nurse-led early rehabilitation program on this basis combines rehabilitation therapy with nursing care, in which the charge nurse provided patients with multifaceted, comprehensive, scientific, and full rehabilitation guidance and care after implementing an individualized assessment of their condition. The functions of early rehabilitation program following surgery are to help the patients after undergoing robot-assisted minimally invasive surgery of the lumbar spine recover quickly. In this study, we analyzed the effect of a nurse-led early rehabilitation program in patients with robot-assisted minimally invasive lumbar degenerative lesions through a prospective study, and on this basis, we discussed improvement of the postoperative early rehabilitation program and nursing model. We present the following article in accordance with the CONSORT reporting checklist (available at https://dx.doi.org/10.21037/ apm-21-2294).

\section{Methods}

\section{Study design and participants}

This is a prospective, open-label randomized clinical trial with 1:1 ratio. Participants did not know which group to be assigned to before randomization. The random numbers were generated followed the centralized allocation, using the same appearance, continuous numbered opaque envelopes. The inclusion criteria for this study were as follows: (I) patients to be treated with minimally invasive lumbar internal fixation after examination and diagnosis; (II) no other serious underlying diseases; (III) voluntary participation. The screening criteria were as follows: (I) poor postoperative general condition; (II) accompanied by severe underlying diseases; (III) osteoarthritis of the extremities resulting in walking impairment; (IV) previous history of mental illness; (V) already participated in other 
Table 1 Comparison of general information between the two groups of participants $\left(\bar{x}_{ \pm s}\right)$

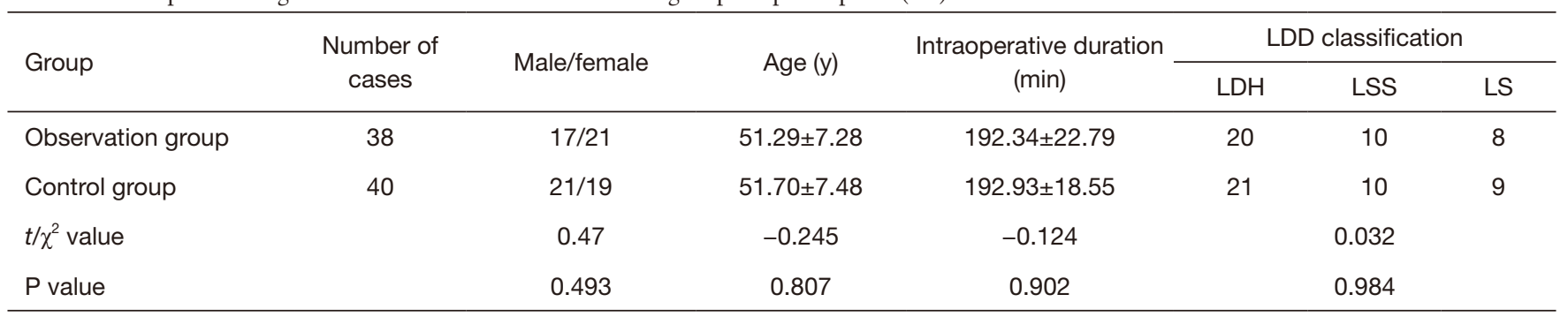

LDD, lumbar degenerative disease; LDH, lumbar disc herniation; LSS, lumbar spinal stenosis; LS, lumbar spondylolisthesis.

clinical trials. The exclusion criteria were the following: (I) not following the early rehabilitation program, including those patients who wanted change his/her groups; (II) cases that did not complete postoperative follow-up and data collection. A total of 80 patients who underwent orthopedic robot-assisted minimally invasive treatment of lumbar degenerative lesions at our hospital between January 2019 and January 2021 were included in this study, and after randomization, there were 40 participants in the observation group initially and 2 cases were excluded midway, and a total of 40 participants in the control group. A nurseled early rehabilitation team provided routine care to the control group participants, while an early rehabilitation program was added to the observation group participants. All procedures performed in this study involving human participants were in accordance with the Declaration of Helsinki (as revised in 2013). The protocol was approved by the Ethics Committee of Sichuan Provincial People's Hospital-Sichuan Academy of Medical Sciences (No. 298, 2019), and all the participants gave informed consent before taking part in the study. There was no difference in the general information between the two groups of participants $(\mathrm{P}>0.05)$, as shown in Table 1.

\section{Research methodology}

\section{Control group}

The routine care model after lumbar spine surgery was implemented, including preoperative fasting for $8 \mathrm{~h}$, preoperative psychological guidance, postoperative assessment of general condition, postoperative guidance of patients' eating, regular axial turning, absolute rest in a flat position, and the plasma drainage tube was removed before getting out of bed.

\section{Observation group}

The early rehabilitation program was added on the basis of the control group.

\section{Construction of early rehabilitation team}

The early rehabilitation team consisted of 21 members, including 5 doctors ( 2 medical team leaders, 2 attending physicians, 1 anesthesiologist), 12 nurses (1 nurse leader, 1 rehabilitation specialist nurse, 2 orthopedic specialist nurses, 8 charge nurses), and in addition, the team was equipped with a rehabilitation therapist, a nutritionist and prosthetic orthopedic technician, and a psychological counselor. Among them, the medical team leader was responsible for developing and implementing the treatment plan; the nurse manager, rehabilitation specialist nurse, and orthopedic specialist nurse were jointly responsible for the implementation of the workflow of the early rehabilitation program and quality control during the implementation process; the charge nurse conducted basic and specialist patient assessments, and together with the attending physician, conducted a comprehensive patient assessment in order to implement the nurse-led early rehabilitation program in a more professional and standardized manner, and to answer patients' questions during the treatment process. The rehabilitation counselor assisted the nurse in implementing the early rehabilitation program and provided training and guidance for any identified weak links; the nutritionist conducted individualized assessment of the patient's nutritional status and selected a suitable diet for the patient; the psychological therapist was responsible for preoperative intervention to reduce patient stress and their postoperative agoraphobia. The orthopedic technician was responsible for making an individualized lumbar support, dynamically adjusting the size of the lumbar brace according to the patient's postoperative condition, and designing the outlet of the plasma drainage tube to facilitate the patient's movement out of bed with the tube in situ.

\section{Nurse-led early rehabilitation program development}

All members of the early rehabilitation team, except the charge nurse, discussed and made decisions based 
Table 2 Nurse-led early rehabilitation program

\begin{tabular}{|c|c|}
\hline Post-operative time & Rehabilitation programs \\
\hline \multirow{2}{*}{ Day of surgery } & (II) Bedside exercises 4 times/d, 10-20 sets/time, each lasting 5-10 min \\
\hline & (III) Clockwise abdominal massage exercises 15 movements/time, 4 times/d \\
\hline \multirow[t]{2}{*}{ Postoperative day 1} & (I) Breathing exercises, bedside exercises, and abdominal massage exercises as on the day of surgery \\
\hline & (III) Double assistance from bedside standing to slow indoor walking $5-10 \mathrm{~m}, 3$ times/d \\
\hline \multirow[t]{2}{*}{ Postoperative day 2} & (I) Breathing exercises 10-15 times/set, bedside exercises $20-30$ times/set, 4 times/d, each lasting 30 min \\
\hline & (II) Wear lumbar support and assist the patient to walk slowly indoors for 3-8 min \\
\hline Postoperative day 3 & (III) Individualized discharge rehabilitation guidance for patients who have had their drains removed \\
\hline \multirow{2}{*}{$\begin{array}{l}\text { Postoperative day } 4- \\
\text { discharge }\end{array}$} & (I) Individualized discharge rehabilitation guidance for patients who have had their drainage tubes removed \\
\hline & $\begin{array}{l}\text { (II) For patients with drainage tubes removed today and those without drainage tubes removed, the } \\
\text { rehabilitation program is the same as at } 3 \text { days after surgery, and the above rehabilitation program is adhered } \\
\text { to until discharge }\end{array}$ \\
\hline
\end{tabular}

Recumbent exercises include ankle pump exercises, straight leg raises, and functional exercises for the quadriceps.

on literature searches (11-18) to develop a nurse-led early postoperative rehabilitation program based on the advantages and characteristics of robot-assisted minimally invasive lumbar degenerative internal fixation combined with the importance of early postoperative rehabilitation exercises (Table 2).

\section{Implementation of a nurse-led early rehabilitation program}

(I) Quality control: first, the nurse manager, rehabilitation specialist nurse, and orthopedic specialist nurse were jointly responsible for the implementation of the workflow of the early rehabilitation program. Second, the rehabilitation therapist and the rehabilitation specialist nurse were made jointly responsible for the training and assessment of the charge nurses, and they were allowed to enter this team only after passing the grade. Third, the department used clinical cases as a template to rehearse this every month, effectively evaluated the early rehabilitation program implemented by the charge nurses, and discussed and rectified any problems to ensure homogeneous implementation of the rehabilitation program. Fourth, the department carries out monthly tests related to the early rehabilitation program, and the charge nurses need to pass the tests.

(II) Preliminary preparation involved the production of the "Manual of rapid postoperative rehabilitation for patients undergoing robot-assisted minimally invasive surgery", which includes the advantages of the third-generation "Tiangui" orthopedic robot, the importance of early postoperative rehabilitation exercises, and the early rehabilitation exercise program with detailed illustrations and notes after discharge from the hospital. We organized team members to shoot a video of the early rehabilitation exercises for patients to learn before and after surgery.

(III) Preoperative preparation: (i) breathing training, also known as abdominal lip retraction breathing: let the abdomen expand slowly, to the patient's maximum tolerance, hold the breath for 3-5 s, and then retract the lips slowly exhale; (ii) through the form of brochures, videos, and on-site teaching, demonstrate and teach patients the bedside exercise movements, 
and strive to standardize them; (iii) a prosthetic orthopedic technician tailor-made a lumbar support for the patient, and demonstrated the correct way to wear it, with the aim of encouraging patients to adapt to it in advance. This involved the innovative setting of a plasma drainage tube outlet on the lumbar support to avoid folding and squeezing of the drainage tube, thus enabling patients to get out of bed early.

(IV) Pain management: patients with LDD endure long standing chronic pain and are likely to resist or refuse to accept an early rehabilitation program due to fear of pain associated with early postoperative rehabilitation exercises (19). To alleviate patients' fears, we used a combination of pre-emptive, ontime, and multimodal analgesia for each patient for the entire pain management process. Nurses and attending physicians adjusted medications according to whether patients felt pain during rehabilitation exercises, thereby improving patients' cooperation with early rehabilitation exercises and alleviating their fear of postoperative exercises.

(V) Precautions: the nurse-led early rehabilitation program should be based on a comprehensive assessment of the patient's condition and systemic status, with the principle of gradual and consistent progress. At the same time, the patient's conditions should be dynamically evaluated, and timely communication with the attending physician and rehabilitation therapist should be made to strive to implement a case-based early rehabilitation program for each patient. At the same time, the charge nurse should know the precautions of this program during the implementation process: (i) the charge nurse performed a holistic patient assessment before implementing the early rehabilitation program and communicated with the attending physician and rehabilitation therapist before implementing the program; (ii) during implementation of the rehabilitation exercise program, the charge nurse closely monitored the patient's vital signs and vigilantly maintained safety protection to prevent the occurrence of bed falls and other adverse events caused by postural hypotension or hypoglycemia; (iii) plasma drainage tubes were properly fixed to prevent spontaneous extubation; (iv) hand hygiene was strictly adhered to during the implementation of interventions to prevent in-hospital cross-infection; (v) communication was maintained with patients throughout the implementation process, paying full attention to their subjective feelings and pain conditions, and providing humanistic care to the greatest extent.

(VI) Psychological guidance: the charge nurse paid attention to the psychological condition of the patient at all times, and if abnormalities were detected, she would contact the psychological therapist promptly to effectively guide the patient together. The charge nurse could use music to soothe the patient's mood and encourage them to share their exercise results with other patients to improve their compliance.

(VII) Follow-up: after a comprehensive assessment of the patient by the charge nurse, attending physician, and rehabilitation therapist before discharge, a customized post-discharge functional exercise plan was formulated. The rehabilitation nurse followed up with the patient every week, including the patient's exercise, brace wearing, medication, wound healing, diet, stool, and so on. They also recorded the patient's individualized rehabilitation goal achievement, provided timely feedback to the patient or the patient's family, and instructed the patient to come to the outpatient clinic for review and followup at 1 month after surgery.

\section{Evaluation indicators}

The primary endpoints of this study were the general postoperative conditions, the scores of daily living ability, the degree of low back pain and functional recovery. (I) Postoperative time to the floor, drainage, and hospitalization; (II) assessment of daily living ability, pain, and functional recovery (3 days and 1 month postoperatively, respectively), in which the Barthel index (BI) was used to evaluate the daily living ability, the visual analogue scale (VAS) was used to evaluate the postoperative pain, and the Oswestry disability index (ODI) was used to evaluate the functional recovery; (III) the patients' compliance with the exercise program on days 1 and 4 after implementation of the exercise program in both groups; and (IV) the incidence of complications in both groups at 1 month after surgery.

\section{Statistical analysis}

Statistical analysis was performed using the software SPSS 


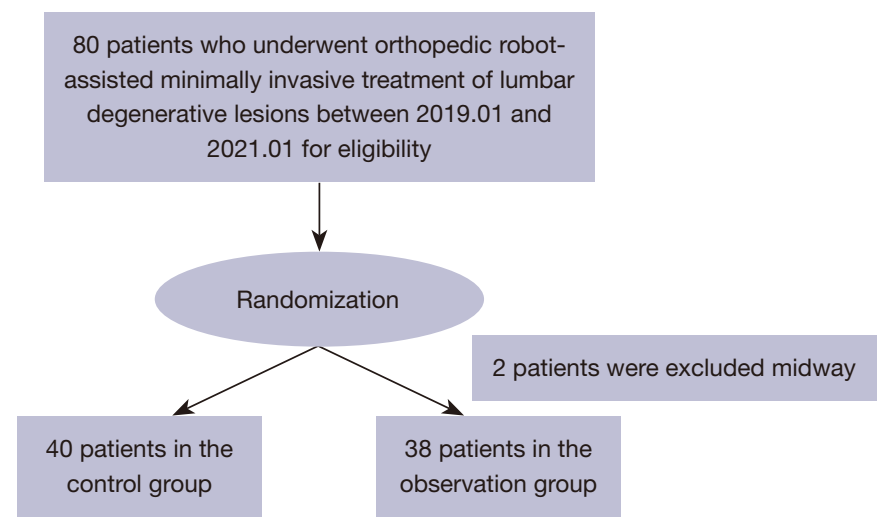

Figure 1 Flow chart.

Table 3 Comparison of postoperative conditions between the two groups of patients $\left(\bar{x}_{ \pm s, \mathrm{~d}}\right)$

\begin{tabular}{lcccc}
\hline Group & Example & Drainage time & Downtime & Length of hospitalization \\
\hline Observation group & 38 & $2.14 \pm 0.40$ & $1.45 \pm 0.37$ & $4.32 \pm 0.70$ \\
Control group & 40 & $2.30 \pm 0.60$ & $2.90 \pm 0.55$ & $4.80 \pm 0.69$ \\
$t$ value & & -1.354 & -13.751 & -3.08 \\
$P$ value & & 0.18 & $<0.001$ & 0.003 \\
\hline
\end{tabular}

Postoperative drainage time, time from placement of surgical plasma drainage tube to its removal; postoperative first time on the floor after surgery, time from the completion of surgery to return to the ward to the time of getting out of bed and standing in lumbar support; postoperative hospitalization time, time from the completion of surgery to discharge.

22.0 IBM Corp., Armonk, NY, USA). The measurement data were expressed as mean \pm standard deviation $(\bar{x} \pm s)$, compared within groups using paired $t$-test, and between groups using independent samples $t$-test; the results of clinical data were expressed as the number of cases and analyzed using the chi-square $\left(\chi^{2}\right)$ test or Fisher's exact test. Statistical significance was considered at $\mathrm{P}<0.05$.

\section{Results}

Comparison of the postoperative conditions of participants in the two groups

Eighty participants who underwent orthopedic robotassisted minimally invasive treatment of lumbar degenerative lesions at our hospital between January 2019 and January 2021 were randomly assigned with 1:1 ratio, 40 participants in the control group without exclusion after randomization, 40 participants in the observation group but excluded 2 patients midway. Finally, 78 patients were analyzed for the primary outcome (Figure 1).

Participants in the observation group were able to move to the floor earlier after surgery $(\mathrm{P}<0.001)$ and their hospital stay was significantly shorter $(\mathrm{P}=0.003)$; there was no significant difference in the postoperative drainage time between the two groups $(\mathrm{P}=0.18)$, as shown in Table 3 .

\section{Comparison of BI, VAS, and ODI scores between two groups of participants}

At 3 days after surgery, the participants in the observation group had significantly better ability to perform daily activities (BI score) than the control group $(\mathrm{P}=0.039)$; their lumbar spine pain (VAS score, $\mathrm{P}=0.028$ ) and functional impairment (ODI score, $\mathrm{P}=0.002$ ) were significantly better than those in the control group; however, there was no significant difference in the three scores between the two groups at 1 month after surgery (all $\mathrm{P}>0.05$ ) (Tables $4-6$ ).

\section{Comparison of days 1 and 4 compliance after implementation of the early rehabilitation program in the two groups}

The compliance at days 1 and 4 after implementation of the 
early rehabilitation program was higher in the observation group than in the control group $(\mathrm{P}<0.001)$, as shown in Table 7 .

Table 4 Comparison of BI scores ( $x \pm s$, points)

\begin{tabular}{lccc}
\hline Group & Example & $\begin{array}{c}\text { Postoperative } \\
\text { day 3 }\end{array}$ & $\begin{array}{c}\text { Postoperative } \\
1 \text { month }\end{array}$ \\
\hline Observation group & 38 & $41.05 \pm 10.54$ & $94.34 \pm 2.89$ \\
Control group & 40 & $34.5 \pm 16.44$ & $90.5 \pm 14.97$ \\
$t$ value & & 2.106 & 1.554 \\
$P$ value & & 0.039 & 0.124 \\
\hline
\end{tabular}

$\mathrm{BI}$, Barthel index.

Table 5 Comparison of VAS scores $(\bar{x} \pm s$, points)

\begin{tabular}{lccc}
\hline Group & Example & $\begin{array}{c}\text { Postoperative } \\
\text { day } 3\end{array}$ & $\begin{array}{c}\text { Postoperative } \\
1 \text { month }\end{array}$ \\
\hline Observation group & 38 & $2.00 \pm 0.57$ & $1.42 \pm 0.55$ \\
Control group & 40 & $2.30 \pm 0.61$ & $1.65 \pm 0.66$ \\
$t$ value & & -2.247 & -1.662 \\
$P$ value & & 0.028 & 0.101 \\
\hline
\end{tabular}

VAS, visual analogue scale.

Table 6 Comparison of ODI scores $(\bar{x} \pm s$, points $)$

\begin{tabular}{lccc}
\hline Group & Example & $\begin{array}{c}\text { Postoperative } \\
\text { day } 3\end{array}$ & $\begin{array}{c}\text { Postoperative } \\
1 \text { month }\end{array}$ \\
\hline Observation group & 38 & $17.47 \pm 1.81$ & $8.71 \pm 1.71$ \\
Control group & 40 & $19.03 \pm 2.34$ & $9.15 \pm 1.44$ \\
$t$ value & & -3.286 & -1.230 \\
$P$ value & & 0.002 & 0.222 \\
\hline
\end{tabular}

ODI, Oswestry disability index.

\section{Comparison of complications between the two groups of participants}

The probability of constipation $(\mathrm{P}=0.043)$ and abdominal distension $(\mathrm{P}=0.012)$ was significantly lower in the observation group within 1 month after surgery, but there was no significant difference in the incidence of other common complications $(\mathrm{P}>0.05)$, as shown in Table 8 .

\section{Discussion \\ The inevitability of implementing a nurse-led early rebabilitation program}

In middle-aged and elderly patients, LDD is a common cause of low back pain, and when conservative treatment fails, surgical treatment is required. The traditional surgical approach has a series of problems such as the slow recovery of body function after surgery and long-term low back pain (20). In recent years, with the promotion of the "Tiangui" orthopedic robot, the implementation of minimally invasive lumbar internal fixation has widely resolved the drawbacks of the traditional surgical approach. The change of surgical modality has also presented new requirements for the corresponding postoperative care.

Rehabilitation treatment can consolidate the therapeutic effect of orthopedic surgery well and accelerate the postoperative recovery of patients. However, at present, the postoperative rehabilitation program related to orthopedic robot-assisted minimally invasive lumbar internal fixation for lumbar degenerative lesions has not been adequately addressed, and there are many problems such as difficulties in team construction, lack of unified early rehabilitation program, lack of charge nurses, and so on. Most hospital rehabilitation work is still led by clinicians and rehabilitation therapists, and nurses provide adjunct assistance (21). The nurse fulfills one of the closest roles in the medical service

Table 7 Comparison of days 1 and 4 compliance after implementation of the early rehabilitation program in the two groups

\begin{tabular}{|c|c|c|c|c|c|c|c|}
\hline Group & $\begin{array}{c}\text { Number of } \\
\text { cases }\end{array}$ & \multicolumn{3}{|c|}{ Day 1} & \multicolumn{3}{|c|}{ Day 4} \\
\hline Observation group & 38 & 25 & 12 & 1 & 23 & 12 & 3 \\
\hline Control group & 40 & 10 & 20 & 10 & 6 & 22 & 12 \\
\hline$\chi^{2}$ value & & \multicolumn{3}{|c|}{15.751} & \multicolumn{3}{|c|}{17.393} \\
\hline
\end{tabular}

Full compliance means that the patient actively completed the exercise content arranged by the charge nurse; partial compliance, supervised completion; non-compliance, unable to cooperate in completion. 
Table 8 Comparison of complication rates between the two groups of participants within 1 month after surgery

\begin{tabular}{lcccccc}
\hline \multirow{2}{*}{ Group } & \multirow{2}{*}{ Example } & \multicolumn{5}{c}{ Common complication } \\
\cline { 3 - 7 } & & Lung infection & Urinary tract infection & Constipation & Bloating & Wound infection \\
\hline Observation group & 38 & 0 & 0 & 5 & 7 & 0 \\
Control group & 40 & 1 & 1 & 13 & 18 & 1 \\
$\chi^{2}$ value & & & 4.107 & 6.321 \\
$P$ value & & $0.513^{\mathrm{a}}$ & $0.513^{\mathrm{a}}$ & 0.043 & 0.012 & $0.513^{\mathrm{a}}$ \\
\hline
\end{tabular}

${ }^{\mathrm{a}}$, the expected number is less than 5 . Fisher's exact test was used.

to patients. In recent years, quality holistic nursing care has been introduced into the front-line clinic, and nurses can fathom the patient's condition most intuitively. At the same time, the establishment of trust and cooperation between nurses and patients means that quality nursing care and clinical treatment have reached a new milestone. With the development of intelligent and precise medical treatment in orthopedics, alongside acceleration of the concept of surgical rehabilitation in the clinic, the theoretical knowledge and professional ability of orthopedic specialist nurses have been further enhanced, and they have assumed leading role in the early rehabilitation program team while encompassing the important duties of assessment, implementation, communication, and observation, and occupying a crucial position in the early rehabilitation program. All members of this team have relevant clinical work experience, have been professionally trained in rehabilitation therapy, and certified by the hospital, having achieved a certain level of competency to meet the particular needs of patients and hospitals regarding post-operative lumbar spine rehabilitation. Unlike the conventional model of traditional postoperative lumbar spine care, the nurse-led early rehabilitation program on this basis combines rehabilitation therapy with nursing care, enabling effective monitoring and control of the patient's condition while focusing on holistic treatment measures for those with degenerative diseases treated by orthopedic robotassisted minimally invasive lumbar, which is in line with the concept of precision nursing and is of great significance to the patient's early recovery.

\section{Science and feasibility of implementing a nurse-led early rehabilitation program}

Previous literature has reported that early rehabilitation exercises can enhance patients' spinal stability, accelerate the repair of local muscles and blood circulation, relieve postoperative lumbar nerve root adhesions, further reduce pain, and provide a basis for further rehabilitation exercises after discharge, enabling rapid patient recovery $(12,14,22)$. In this study, we showed that among patients who underwent orthopedic robotic-assisted minimally invasive lumbar internal fixation surgery, the average time to start moving on the ground was 1.45 days after surgery for those who received the nurse-led early rehabilitation program, while the average time to start moving on the ground was 2.90 days after surgery for those who received conventional care, suggesting that the nurse-led early rehabilitation program accelerated patients' early postoperative movement on the ground. The postoperative hospital stay of participants in the early rehabilitation group was also significantly shorter than that of those in the conventional care group, fully confirming that implementation of the nurse-led early rehabilitation in the postoperative period was sufficient to hasten patients' postoperative recovery, which is consistent with previous reports (12). In addition, patients who undertook the early rehabilitation program had a significantly higher ability to perform daily activities 3 days after surgery than those who received conventional care, and their VAS and ODI scores were significantly lower $(\mathrm{P}<0.05)$, indicating that the nurse-led early rehabilitation program helped to improve patients' living ability in the early postoperative period, alleviate their early postoperative pain levels, and reduce the application of postoperative analgesics and analgesic pumps. In addition, participants in the observation group were not prone to constipation and bloating, which also suggested that implementation of the nurse-led early rehabilitation program in the postoperative period could effectively reduce the occurrence of postoperative gastrointestinal complications.

\section{Implementation of a nurse-led early recovery program can reduce patient agoraphobia}

Patients with LDD experience from lasting chronic pain 
and are likely to resist or reject an early rehabilitation program due to a fear of pain associated with early postoperative rehabilitation exercises (19). In this study, a nurse-led early rehabilitation program was adopted, in which the charge nurse provided patients with multifaceted, comprehensive, scientific, and full rehabilitation guidance and care after implementing an individualized assessment of their condition. In terms of compliance, that of patients in the observation group at $\mathrm{d} 1$ and $\mathrm{d} 4$ was higher than that of patients in the control group $(\mathrm{P}<0.001)$, suggesting that the nurse-led aspect of early rehabilitation was instrumental in gaining patients' trust, helping patients establish confidence in early recovery, reducing patients' agoraphobia, and enhancing patients' awareness of self-exercise.

\section{Suggestions for implementing a nurse-led early recovery program after minimally invasive lumbar internal fixation}

We suggested that those who experienced minimally invasive lumbar internal fixation should receive a nurseled early rehabilitation program as early as possible. In addition, although the nurse fulfills one of the closest roles in the medical service to patients, they should timely communication with the attending physician and each patient to dynamically assess the patient's condition and systemic status, to strive to implement a case-based early rehabilitation program for each patient.

\section{Conclusions}

The implementation of a nurse-led early rehabilitation program in patients undergoing orthopedic robotassisted minimally invasive lumbar internal fixation not only improves patient compliance, shortens patients' postoperative time to the floor, and actively promotes rapid postoperative recovery, but also reduces the occurrence of postoperative gastrointestinal complications, bringing both immediate and long-term potential clinical benefits to patients. In addition, our study also showcased the valuable nursing experience to our colleagues, effectively enhancing the role of nurses in early rehabilitation exercises and improving their professional value. As this study was a single-center study, it is only representative of the functional rehabilitation exercises of patients undergoing robotic minimally invasive lumbar internal fixation surgery in our orthopedic department, and further studies are needed on this basis to obtain more scientific findings.

\section{Acknowledgments}

Funding: None.

\section{Footnote}

Reporting Checklist: The authors have completed the CONSORT guideline checklist. Available at https://dx.doi. org/10.21037/apm-21-2294

Trial Protocol: Available at https://dx.doi.org/10.21037/apm21-2294

Data Sharing Statement: Available at https://dx.doi. org/10.21037/apm-21-2294

Conflicts of Interest: All authors have completed the ICMJE uniform disclosure form (available at https://dx.doi. org/10.21037/apm-21-2294). The authors have no conflicts of interest to declare.

Etbical Statement: The authors are accountable for all aspects of the work in ensuring that questions related to the accuracy or integrity of any part of the work are appropriately investigated and resolved. All procedures performed in this study involving human participants were in accordance with the Declaration of Helsinki (as revised in 2013). The study protocol was approved by the Medical Ethics Committee of Sichuan Provincial People's HospitalSichuan Academy of Medical Sciences (No. 298, 2019) and all the participants gave informed consent before taking part in the study.

Open Access Statement: This is an Open Access article distributed in accordance with the Creative Commons Attribution-NonCommercial-NoDerivs 4.0 International License (CC BY-NC-ND 4.0), which permits the noncommercial replication and distribution of the article with the strict proviso that no changes or edits are made and the original work is properly cited (including links to both the formal publication through the relevant DOI and the license). See: https://creativecommons.org/licenses/by-nc-nd/4.0/.

\section{References}

1. Kao YC, Chen JY, Chen HH, et al. The association between depression and chronic lower back pain from disc degeneration and herniation of the lumbar spine. 
Int J Psychiatry Med 2021. [Epub ahead of print]. doi: 10.1177/00912174211003760.

2. Yolcu YU, Moinuddin FM, Wahood W, et al. Use of regenerative treatments in treatment of lumbar degenerative disc disease: a systematic review. Clin Neurol Neurosurg 2020;195:105916.

3. Jang SH, Chang MC. Follow-up of at least five years after lumbar transforaminal epidural steroid injection for radicular pain due to lumbar disc herniation. Ann Palliat Med 2020;9:116-8.

4. Czabanka M, Thomé C, Ringel F, et al. Operative treatment of degenerative diseases of the lumbar spine. Nervenarzt 2018;89:639-47.

5. Momin AA, Steinmetz MP. Evolution of minimally invasive lumbar spine surgery. World Neurosurg 2020;140:622-6.

6. Kochanski RB, Lombardi JM, Laratta JL, et al. Imageguided navigation and robotics in spine surgery. Neurosurgery 2019;84:1179-89.

7. Ghasem A, Sharma A, Greif DN, et al. The arrival of robotics in spine surgery: a review of the literature. Spine (Phila Pa 1976) 2018;43:1670-7.

8. Epstein NE, Hollingsworth RD. Nursing review of diagnosis and treatment of lumbar degenerative spondylolisthesis. Surg Neurol Int 2017;8:246.

9. Tarnanen SP, Neva MH, Häkkinen K, et al. Neutral spine control exercises in rehabilitation after lumbar spine fusion. J Strength Cond Res 2014;28:2018-25.

10. Nielsen PR, Andreasen J, Asmussen M, et al. Costs and quality of life for prehabilitation and early rehabilitation after surgery of the lumbar spine. BMC Health Serv Res 2008;8:209.

11. Skrobot W, Liedtke E, Krasowska K, et al. Early rehabilitation program and vitamin $\mathrm{D}$ supplementation improves sensitivity of balance and the postural control in patients after posterior lumbar interbody fusion: a randomized trial. Nutrients 2019;11:2202.

12. Macki M, Zakaria HM, Massie LW, et al. The effect of physical therapy on time to discharge after lumbar interbody fusion. Clin Neurol Neurosurg 2020;197:106157.

Cite this article as: $\mathrm{He}$ W, Wang Q, Hu J, Lin S, Zhang K, Wang F, Xu C, Li F, Xiao J, Li X, Tang F. A randomized trial on the application of a nurse-led early rehabilitation program after minimally invasive lumbar internal fixation. Ann Palliat Med 2021;10(9):9820-9829. doi: 10.21037/apm-21-2294
13. Oosterhuis T, Costa LO, Maher CG, et al. Rehabilitation after lumbar disc surgery. Cochrane Database Syst Rev 2014;(3):CD003007.

14. Tarnanen S, Neva MH, Dekker J, et al. Randomized controlled trial of postoperative exercise rehabilitation program after lumbar spine fusion: study protocol. BMC Musculoskelet Disord 2012;13:123.

15. Ozkara GO, Ozgen M, Ozkara E, et al. Effectiveness of physical therapy and rehabilitation programs starting immediately after lumbar disc surgery. Turk Neurosurg 2015;25:372-9.

16. Kernc D, Strojnik V, Vengust R. Early initiation of a strength training based rehabilitation after lumbar spine fusion improves core muscle strength: a randomized controlled trial. J Orthop Surg Res 2018;13:151.

17. Santana-Ríos JS, Chívez-Arias DD, Coronado-Zarco R, et al. Postoperative treatment for lumbar disc herniation during rehabilitation. Systematic review. Acta Ortop Mex 2014;28:113-24.

18. Marchand AA, O'Shaughnessy J, Châtillon CÉ, et al. Current practices in lumbar surgery perioperative rehabilitation: a scoping review. J Manipulative Physiol Ther 2016;39:668-92.

19. Jabłońska R, Ślusarz R, Królikowska A, et al. Depression, social factors, and pain perception before and after surgery for lumbar and cervical degenerative vertebral disc disease. J Pain Res 2017;10:89-99.

20. Tian F, Tu LY, Gu WF, et al. Percutaneous versus open pedicle screw instrumentation in treatment of thoracic and lumbar spine fractures: a systematic review and metaanalysis. Medicine (Baltimore) 2018;97:e12535.

21. Munday J, Higgins N, Mathew S, et al. Nurse-led randomized controlled trials in the perioperative setting: a scoping review. J Multidiscip Healthc 2020;13:647-60.

22. Angelini E, Baranto A, Brisby H, et al. Healthcare practitioners' experiences of postoperative pain management in lumbar spine surgery care-A qualitative study. J Clin Nurs 2020;29:1662-72.

(English Language Editor: J. Jones) 